Genetics Research

cambridge.org/grh

\section{Research Paper}

Cite this article: Nguyen-Dumont $\mathrm{T}$ et al (2020). Genetic testing in Poland and Ukraine: should comprehensive germline testing of $B R C A 1$ and $B R C A 2$ be recommended for women with breast and ovarian cancer? Genetics Research 102, e6, 1-7. https://doi.org/ $10.1017 / \mathrm{S} 0016672320000075$

Received: 13 March 2020

Revised: 13 June 2020

Accepted: 15 June 2020

\section{Keywords:}

$B R C A 1 ; B R C A 2$; breast cancer; founder mutations; genetic susceptibility; genetic testing; ovarian cancer

Author for correspondence:

Professor Melissa C. Southey,

E-mail: melissa.southey@monash.edu

\title{
Genetic testing in Poland and Ukraine: should comprehensive germline testing of BRCA1 and $B R C A 2$ be recommended for women with breast and ovarian cancer?
}

\begin{abstract}
Tu Nguyen-Dumont ${ }^{1,2}$ (D), Pawel Karpinski ${ }^{3}$, Maria M. Sasiadek ${ }^{3}$, Hayane Akopyan ${ }^{4,5}$, Jason A. Steen ${ }^{1}$, Derrick Theys ${ }^{1}$, Fleur Hammet ${ }^{1,2}$, Helen Tsimiklis ${ }^{1,2}$, Daniel J. Park ${ }^{2,6}$ (D), Bernard J. Pope ${ }^{1,6,7}$ (D), Ryszard Slezak ${ }^{3}$, Agnieszka Stembalska ${ }^{3}$, Karolina Pesz ${ }^{3}$, Nataliya Kitsera ${ }^{4}$, Aleksandra Siekierzynska ${ }^{8}$, Melissa C. Southey ${ }^{1,2,9}$ (D) and Aleksander Myszka ${ }^{5}$

${ }^{1}$ Precision Medicine, School of Clinical Sciences at Monash Health, Monash University, Clayton, Australia; ${ }^{2}$ Department of Clinical Pathology, The University of Melbourne, Melbourne, Australia; ${ }^{3}$ Department of Genetics, Wroclaw Medical University, Wroclaw, Poland; ${ }^{4}$ Institute of Hereditary Pathology of National Academy of Medical Sciences, Lviv, Ukraine; ${ }^{5}$ Institute of Medical Sciences, University of Rzeszow, Rzeszow, Poland; ${ }^{6}$ Melbourne Bioinformatics, The University of Melbourne, Melbourne, Australia; ${ }^{7}$ Colorectal Oncogenomics Group, Department of Clinical Pathology, The University of Melbourne, Melbourne, Australia; ${ }^{8}$ Department of Biotechnology and Plant Physiology, University of Rzeszow, Rzeszow, Poland and ${ }^{9}$ Cancer Epidemiology Division, Cancer Council Victoria, Melbourne, VIC 3004, Australia
\end{abstract}

\begin{abstract}
Purpose. To characterize the spectrum of $B R C A 1$ and $B R C A 2$ pathogenic germline variants in women from south-west Poland and west Ukraine affected with breast or ovarian cancer. Testing in women at high risk of breast and ovarian cancer in these regions is currently mainly limited to founder mutations.

Methods. Unrelated women affected with breast and/or ovarian cancer from Poland $(n=337)$ and Ukraine $(\mathrm{n}=123)$ were screened by targeted sequencing. Excluded from targeted sequencing were 34 Polish women who had previously been identified as carrying a founder mutation in $B R C A 1$. No prior testing had been conducted among the Ukrainian women. Thus, this study screened BRCA1 and BRCA2 in the germline DNA of 426 women in total.

Results. We identified 31 and 18 women as carriers of pathogenic/likely pathogenic (P/LP) genetic variants in $B R C A 1$ and $B R C A 2$, respectively. We observed five BRCA1 and eight BRCA2 P/LP variants (13/337, 3.9\%) in the Polish women. Combined with the $34 / 337$ (10.1\%) founder variants identified prior to this study, the overall P/LP variant frequency in the Polish women was thus $14 \%$ (47/337). Among the Ukrainian women, 16/123 (13\%) women were identified as carrying a founder mutation and 20/123 (16.3\%) were found to carry non-founder P/LP variants (10 in BRCA1 and 10 in BRCA2).

Conclusions. These results indicate that genetic testing in women at high risk of breast and ovarian cancer in Poland and Ukraine should not be limited to founder mutations. Extended testing will enhance risk stratification and management for these women and their families.
\end{abstract}

\section{Introduction}

Women who carry a pathogenic mutation in BRCA1 or BRCA2 are at increased risk of developing breast and ovarian cancer. Kuchenbaecker et al. estimated the cumulative breast cancer risk to age 80 years to be $72 \%$ (95\% confidence interval $(\mathrm{CI})=65-79 \%)$ and $69 \%(95 \% \mathrm{CI}=$ 61-77\%) for BRCA1 and BRCA2 pathogenic variant carriers, respectively (Kuchenbaecker et al., 2017). For ovarian cancer, the cumulative cancer risk to age 80 years is estimated to be $44 \%(95 \% \mathrm{CI}=36-53 \%)$ and $17 \%(95 \% \mathrm{CI}=11-25 \%)$ for BRCA1 and BRCA2 pathogenic variant carriers, respectively (Kuchenbaecker et al., 2017).

In many countries, genetic testing for BRCA1 and BRCA2 has shown clear clinical utility and validity. Evidence-based best practice guidelines are available to inform the clinical management of women who carry a $B R C A 1$ or $B R C A 2$ pathogenic variant. These guidelines support personalized risk assessment, targeted treatment regimens and informed decision-making about the use of risk-reducing medications, bilateral salpingo-oophorectomy, mammography, risk-reducing mastectomy, magnetic resonance imaging and other screening modalities.

It is well established that, as a consequence of a founder effect, different ethnic and geographical regions can have different $B R C A 1$ and $B R C A 2$ mutation spectra and prevalence rates. 
Table 1. Reported prevalence of $B R C A 1$ founder mutations in the Polish population.

\begin{tabular}{|c|c|c|c|c|c|}
\hline HGVS_cDNA $^{a}$ & HGVS_p & $\mathrm{BIC}^{b}$ & MAF EXAC & MAF Polish population & References \\
\hline c.5266dup & p.(Gln1756ProfsTer74) & 5382insC & 0.00016 & $1.7 \times 10^{-3}$ to $3.5 \times 10^{-3}$ & Gorski et al. (2005); Brozek et al. (2011) \\
\hline c. $181 \mathrm{~T}>\mathrm{G}$ & p.(Cys61Gly) & $300 \mathrm{~T}>\mathrm{G}$ & $5.64 \mathrm{e}-05$ & $0.5 \times 10^{-3}$ to $0.8 \times 10^{-3}$ & Gorski et al. (2005); Brozek et al. (2011) \\
\hline c.4035del & p.(Glu1346LysfsTer20) & 4153delA & $3.68 \mathrm{e}-05$ & $0.2 \times 10^{-3}$ & Gorski et al. (2005) \\
\hline c.68_69del & p.(Glu23ValfsTer17) & 185delAG & 0.000406 & $<0.1 \times 10^{-3}$ & Brozek et al. (2011) \\
\hline
\end{tabular}

${ }^{a}$ Variant nomenclature according to the Human Genome Variation Society (HGVS). Transcript sequences is BRCA1: NM 007294.3.

${ }^{b}$ Variant nomenclature according to the Breast Cancer Information Core (BIC) (https://research.nhgri.nih.gov/bic).

${ }^{c}$ Minor allele frequency (MAF) in the ExAC database, in the non-Finnish European population minus The Cancer Genome Atlas (Lek et al., 2016).

Cybulski et al. recently reported on the mutation spectrum in $B R C A 1, B R C A 2$ and other genes associated, or putatively associated, with increased risk of breast cancer in 1018 probands from multiple-case breast cancer families from Poland. In their study, three founder mutations were identified with high prevalence: BRCA1:c.5266dup (20\%, 204/1018), BRCA1:c.181T>G $(8.3 \%, 84 / 1018)$ and BRCA1:c.4035del $(1.5 \%, 15 / 1018)$. Other mutations reported at lower prevalence $(\leq 1.0 \%)$ in the 1108 familial breast cancer cases included BRCA1:c.3700_3704del (1.0\% 10/1018), BRCA1:c.68_69del (0.9\%, 9/1018), BRCA1: c.5251C $>\mathrm{T}(0.6 \%, 6 / 1018)$ and BRCA1:c.5346G>A $(0.5 \%, 5 /$ 1108) (Cybulski et al., 2019). Recurrent mutations were also reported in BRCA2 by Cybulski et al., all at a prevalence of $0.5 \%$ or below in the Polish women with familial breast cancer (Cybulski et al., 2019).

The BRCA1 mutations c.5266dupC and c.4035delA have been confirmed by haplotype analysis as founder mutations in Eastern Europe (Hamel et al., 2011; Janavicius et al., 2013). BRCA1: c.181T>G mutation carriers of Polish and Jewish ancestry have been shown to present the same haplotype (Kaufman et al., 2009). Haplotype analysis of BRCA1:c.68_69delAG showed a common haplotype among Ashkenazi Jews (Laitman et al., 2013). Currently, genetic testing in the Polish population mainly relies on testing of the founder mutations. Comprehensive BRCA1 and $B R C A 2$ genetic testing could, however, identify more women with pathogenic variants, thus leading to improved cancer prevention for more women at high risk of breast and ovarian cancer.

Participants in this study were unrelated women affected with breast or ovarian cancer from south-west Poland and west Ukraine. The Polish participants had previously been genotyped for BRCA1:c.5266dup, BRCA1:c.181T>G, BRCA1:c.4035del, BRCA1:c.68_69del and BRCA2:c.5946del. Thirty-four women identified as carrying one of these mutations were excluded from further testing in this study. Participants recruited in Ukraine had not undergone prior genetic testing and have thus all been included in the genetic testing reported in this study.

\section{Materials and methods}

\subsection{Study participants}

The women participating in this study were unrelated women diagnosed with breast and/or ovarian cancer recruited after or during oncological treatment from Wroclaw Medical University, Lower Silesia, Poland, between 2004 and 2008, or Lviv State Oncology Regional Treatment and Diagnostic Center, Lviv, Ukraine, between 2004 and 2010, as described previously (Myszka et al., 2018). The Polish cohort consisted of 238 women affected with breast cancer, 95 women affected with ovarian cancer and 4 women affected with breast and ovarian cancer. Of the 242 women with breast cancer, 95 had hereditary breast cancer, 18 had familial breast cancer and 125 were sporadic cases, according to the criteria described by Berliner et al. (2007). Of the 95 Polish women with ovarian cancer, 28 had hereditary ovarian cancer, 10 had familial ovarian cancer and 57 were sporadic ovarian cancer cases. All four women with breast and ovarian cancers met the criteria for hereditary disease. The Polish cohort thus consisted of 337 women, all of whom had previously been genotyped for four mutations in BRCA1 (c.5266dup, c.181T>G, c.4035del, c.68_69del) (Table 1) and one mutation in BRCA2 (c.5946delT). Thirty-four women were identified as carriers of one of these BRCA1 founder mutations and were thus not included in the targeted sequence screening described in this study. No BRCA2 c.5946delT carrier was observed.

The Ukrainian cohort consisted of 112 women with breast cancer, 10 women with ovarian cancer and 1 woman with breast and ovarian cancer. Seventy-three women affected with breast cancer met the hereditary cancer criteria and 38 women met the familial cancer criteria. For one Ukrainian participant with breast cancer, insufficient information was available to classify her cancer. Of the women with ovarian cancer, six had hereditary ovarian cancer and four had familial ovarian cancer. The woman with breast and ovarian cancer met the hereditary cancer criteria. There had been no previous testing for mutations in BRCA1 and $B R C A 2$ conducted in the Ukrainian participants.

All participants provided informed consent for participation in this research programme, which was approved by the Commission of Bioethics of the Institute of Hereditary Pathology of the National Academy of Medical Sciences of Ukraine, the Ethics Committee of Wroclaw Medical University (Poland), the Ethics Committee of the University of Rzeszow (Poland) and the University of Melbourne Human Research Ethics Committee (Australia).

\subsection{Mutation screening}

Amplicon-based massively parallel sequencing of the proteincoding regions and proximal intron-exon junctions of BRCA1 (NM_007294.3) and BRCA2 (NM_000059.3) was performed using lymphocyte-derived germline DNA and the Hi-Plex protocol (Nguyen-Dumont et al., 2015). All oligonucleotides were synthesized by Integrated DNA Technologies (Coralville, IA, USA). Gene-specific primers and adapter primers were purified to standard desalting and high-performance liquid chromatography grade, respectively. All oligonucleotide sequences are available upon request. Massively parallel sequencing (150 bp paired-end) was performed on the MiSeq system (Illumina, San Diego, CA, USA). Mapping to the human reference build GRCh37 was performed using bwa-mem 0.7.17 (Li \& Durbin, 
Table 2. Pathogenic and likely pathogenic ${ }^{a} B R C A 1$ and BRCA2 mutations carriers identified in 460 women affected with breast or ovarian cancer in south-west Poland and west Ukraine.

\begin{tabular}{|c|c|c|c|c|c|c|c|c|c|}
\hline \multirow[b]{2}{*}{ Gene } & \multirow[b]{2}{*}{ Variant type } & \multirow[b]{2}{*}{ HGVS_c $c^{b}$} & \multirow[b]{2}{*}{ HGVS_p ${ }^{b}$} & \multirow[b]{2}{*}{ Classification $^{c}$} & \multicolumn{5}{|c|}{ Number of carriers } \\
\hline & & & & & Total & $\mathrm{Pol}^{d}$ & $\mathrm{Ukr}^{d}$ & $\mathrm{BC}^{e}$ & $\mathrm{OC}^{\mathrm{e}}$ \\
\hline \multirow[t]{16}{*}{$B R C A 1^{f}$} & \multirow[t]{2}{*}{ Nonsense } & c. $5251 C>T$ & p.Arg1751Ter & Pathogenic & 1 & 1 & 0 & 1 & 0 \\
\hline & & c. $5346 \mathrm{G}>\mathrm{A}$ & p.Trp1782Ter & Pathogenic & 1 & 1 & 0 & 1 & 0 \\
\hline & \multirow[t]{12}{*}{ Frameshift } & c.68_69del $\left.\right|^{g}$ & p.Glu23ValfsTer17 & Pathogenic & 3 & 1 & 2 & 2 & 1 \\
\hline & & c.374dup & p.GIn126ProfsTer16 & No data & 1 & 1 & 0 & 0 & 1 \\
\hline & & c.843_846del & p.Ser282TyrfsTer15 & Pathogenic & 1 & 1 & 0 & 1 & 0 \\
\hline & & c.844_850dup & p.Gln284LeufsTer5 & Pathogenic & 1 & 0 & 1 & 0 & 1 \\
\hline & & c.1510del & p.Arg504ValfsTer28 & Pathogenic & 1 & 0 & 1 & 1 & 0 \\
\hline & & c.1612_1616del & p.GIn538GlyfsTer11 & Pathogenic & 1 & 1 & 0 & 0 & 1 \\
\hline & & c.2217dup ${ }^{h}$ & p.Val740SerfsTer3 & Pathogenic & 1 & 0 & 1 & 1 & 0 \\
\hline & & c.2291_2292del & p.Val764GlyfsTer3 & No data & 1 & 0 & 1 & 1 & 0 \\
\hline & & c. $4035 \mathrm{del}^{g}{ }^{g}$ & p.Glu1346LysfsTer20 & Pathogenic & 2 & 2 & 0 & 0 & 2 \\
\hline & & c.5030_5033del & p.Thr1677llefsTer2 & Pathogenic & 3 & 0 & 3 & 2 & 1 \\
\hline & & c.5177_5180del & p.Arg1726LysfsTer3 & Pathogenic & 2 & 0 & 2 & 2 & 0 \\
\hline & & c.5266dup ${ }^{g}$ & p.GIn1756ProfsTer74 & Pathogenic & 35 & 24 & 11 & 25 & 12 \\
\hline & Splice donor & c. $4357+1 \mathrm{G}>\mathrm{C}$ & - & No data & 1 & 0 & 1 & 1 & 0 \\
\hline & Missense & c. $181 \mathrm{~T}>\mathrm{G}^{g}$ & p.Cys61Gly & Pathogenic & 10 & 7 & 3 & 4 & 6 \\
\hline \multirow[t]{14}{*}{$B R C A 2^{f}$} & Nonsense & c.3075_3076delinsTT & p.Lys1025_Lys1026delinsAsnTer & Pathogenic & 3 & 3 & 0 & 0 & 3 \\
\hline & & c.5857G>T & p.Glu1953Ter & Pathogenic & 1 & 1 & 0 & 1 & 0 \\
\hline & & c. $7721 \mathrm{G}>\mathrm{A}$ & p.Trp2574Ter & Pathogenic & 1 & 0 & 1 & 1 & 0 \\
\hline & & c. $8623 \mathrm{G}>\mathrm{T}$ & p.Glu2875Ter & No data & 1 & 1 & 0 & 1 & 0 \\
\hline & Frameshift & c.2945del & p.lle982AsnfsTer9 & Pathogenic & 1 & 0 & 1 & 1 & 0 \\
\hline & & c.5205_5208del & p.GIn1736IlefsTer4 & Pathogenic & 1 & 1 & 0 & 1 & 0 \\
\hline & & c.6315_6318del & p.Pro2107ValfsTer11 & No data & 1 & 1 & 0 & 1 & 0 \\
\hline & & c.6405_6409del & p.Asn2135LysfsTer3 & Pathogenic & 1 & 0 & 1 & 1 & 0 \\
\hline & & c.6408_6414del & p.Asn2137LysfsTer29 & Pathogenic & 1 & 0 & 1 & 1 & 0 \\
\hline & & c.7069_7070del & p.Leu2357ValfsTer2 & Pathogenic & 1 & 0 & 1 & 1 & 0 \\
\hline & & c.9097dup & p.Thr3033AsnfsTer11 & Pathogenic & 1 & 0 & 1 & 1 & 0 \\
\hline & & c.9253dup & p.Thr3085AsnfsTer26 & Pathogenic & 1 & 1 & 0 & 1 & 0 \\
\hline & & c.10095delinsGAATTATATCT & p.Ser3366AsnfsTer4 & Not yet reviewed & 1 & 0 & 1 & 1 & 0 \\
\hline & Splice donor & c. $475+1 G>T$ & - & No data & 3 & 0 & 3 & 3 & 0 \\
\hline
\end{tabular}

${ }^{a}$ Genetic variants in BRCA1 and BRCA2 that are classified as pathogenic by the expert panel Evidence-based Network for the Interpretation of Germline Mutant Alleles (ENIGMA) (Spurdle et al., 2012) as reported on the BRCA Exchange portal (http:// brcaexchange.org), unreported truncating variants and variants occurring in consensus splice sites.

Variant nomenclature based on +1 as A of ATG start codon, according to the Human Genome Variation Society (HGVS), HGVS_c for coding DNA and HGVS_p for protein variants.

Classification according to the ENIGMA expert panel (Spurdle et al., 2012), available from the BRCA Exchange portal (http://brcaexchange.org).

Pol = Polish; Ukr = Ukrainian

$\mathrm{BC}=$ breast cancer; $\mathrm{OC}=$ ovarian cancer. Some women were diagnosed with both $\mathrm{BC}$ and $\mathrm{OC}$.

Transcript sequences are BRCA1: NM_007294.3 and BRCA2: NM_00059.3.

Founder mutation - identified via targeted sequencing (this study) or via Sanger sequencing (prior testing) (Table 1).

No DNA was available for validation by Sanger sequencing. 
Table 3. Clinical characteristics of pathogenic and likely pathogenic ${ }^{a} B R C A 1$ and $B R C A 2$ variant carriers, identified from 460 women affected with breast or ovarian cancer in south-west Poland and west Ukraine.

\begin{tabular}{|c|c|c|c|c|c|c|}
\hline Cohort & Cancer type & $\begin{array}{c}\text { Cancer } \\
\text { classification }^{b}\end{array}$ & $\begin{array}{l}\text { Studied } \\
\text { cases }\end{array}$ & $\begin{array}{l}\text { Carriers of a } \\
\text { founder mutation }^{c}\end{array}$ & $\begin{array}{c}\text { Carriers of a non-founder } \\
\text { P/LP variant }{ }^{a}\end{array}$ & $\begin{array}{l}\text { Carriers of any } \\
\mathrm{P} / \mathrm{LP} \text { variant }^{a}\end{array}$ \\
\hline \multirow[t]{10}{*}{ Polish } & Breast & Hereditary & 95 & $11(11.6 \%)$ & $4(4.2 \%)$ & $15(15.8 \%)$ \\
\hline & & Familial & 18 & $1(5.5 \%)$ & $1(5.5 \%)$ & $2(11.1 \%)$ \\
\hline & & Sporadic & 125 & $1(0.8 \%)$ & $2(1.6 \%)$ & $3(2.4 \%)$ \\
\hline & & Total & 238 & $13(5.5 \%)$ & $7(2.5 \%)$ & $20(8.4 \%)$ \\
\hline & Ovarian & Hereditary & 28 & $17(60.7 \%)$ & $2(7.1 \%)$ & $19(67.8 \%)$ \\
\hline & & Familial & 10 & 0 & $3(30 \%)$ & $3(30 \%)$ \\
\hline & & Sporadic & 57 & $1(1.7 \%)$ & 0 & $1(1.7 \%)$ \\
\hline & & Total & 95 & $18(18.9 \%)$ & $5(5.3 \%)$ & $23(24.2 \%)$ \\
\hline & Breast and ovarian & Hereditary & 4 & $3(75 \%)$ & $1(25 \%)$ & $4(100 \%)$ \\
\hline & All Polish cases & & 337 & $34(10.1 \%)$ & $13(3.9 \%)$ & $47(13.9 \%)$ \\
\hline \multirow[t]{11}{*}{ Ukrainian } & Breast & Hereditary & 73 & $12(16.4 \%)$ & $14(19.2 \%)$ & $26(35.1 \%)$ \\
\hline & & Familial & 38 & $2(5.3 \%)$ & $4(10.5 \%)$ & $6(15.8 \%)$ \\
\hline & & Sporadic & 0 & - & - & - \\
\hline & & Unknown & 1 & 0 & 1 & 1 \\
\hline & & Total & 112 & $14(12.5 \%)$ & $19(15.4 \%)$ & $33(29.5 \%)$ \\
\hline & Ovarian & Hereditary & 6 & $1(16.7 \%)$ & $1(16.7 \%)$ & $2(33.3 \%)$ \\
\hline & & Familial & 4 & 0 & 0 & 0 \\
\hline & & Sporadic & 0 & - & - & - \\
\hline & & Total & 10 & $1(0.9 \%)$ & $1(0.9 \%)$ & $2(18.2 \%)$ \\
\hline & Breast and ovarian & Hereditary & 1 & 1 & 0 & $1(100 \%)$ \\
\hline & All Ukrainian cases & & 123 & $16(13 \%)$ & $20(16.3 \%)$ & $36(29.3 \%)$ \\
\hline \multirow[t]{11}{*}{ Polish and Ukrainian } & Breast & Hereditary & 168 & $23(13.7 \%)$ & $18(10.7 \%)$ & $41(24.4 \%)$ \\
\hline & & Familial & 56 & $3(5.4 \%)$ & $5(8.9 \%)$ & $8(14.3 \%)$ \\
\hline & & Sporadic & 125 & $1(0.8 \%)$ & $2(1.6 \%)$ & $3(2.4 \%)$ \\
\hline & & Unknown & 1 & 0 & 1 & 1 \\
\hline & & Total & 350 & $27(7.7 \%)$ & $26(7.4 \%)$ & $53(15.1 \%)$ \\
\hline & Ovarian & Hereditary & 34 & $18(52.9 \%)$ & $3(8.8 \%)$ & $21(61.8 \%)$ \\
\hline & & Familial & 14 & 0 & $3(21.4 \%)$ & $3(21.4 \%)$ \\
\hline & & Sporadic & 57 & $1(1.8 \%)$ & 0 & $1(1.8 \%)$ \\
\hline & & Total & 105 & $19(18.1 \%)$ & $6(5.7 \%)$ & $25(23.8 \%)$ \\
\hline & Breast and ovarian & Hereditary & 5 & $4(80 \%)$ & $1(20 \%)$ & $5(100 \%)$ \\
\hline & Total cases & & 460 & $50(10.9 \%)$ & $33(7.2 \%)$ & $83(18.0 \%)$ \\
\hline
\end{tabular}

${ }^{a}$ Genetic variants in BRCA1 and BRCA2 that are classified as pathogenic/likely pathogenic (P/LP) by the expert panel Evidence-based Network for the Interpretation of Germline Mutant Alleles (ENIGMA) (Spurdle et al., 2012) as reported on the BRCA Exchange portal (http://brcaexchange.org), unreported truncating variants and variants occurring in consensus splice sites.

${ }^{b}$ Classification as per Berliner et al. (2007).

${ }^{c}$ Founder mutations in Table 1.

2009). Variant calling was performed using the Java version of VarDict in single-sample, amplicon mode (Lai et al., 2016).

\subsection{Annotation and classification of variants}

Variants were annotated using VEP (v.90) and loaded into GEMINI, according to the authors' recommendations (Paila et al., 2013; McLaren et al., 2016). Classification of genetic variants in BRCA1 and BRCA2 was then performed in accordance with the Evidence-based Network for the Interpretation of Germline Mutant Alleles (ENIGMA) consortium's recommendations (Spurdle et al., 2012). Pathogenicity calls were retrieved from the BRCA Exchange portal (http://brcaexchange.org; accessed 17 June 2019). When no information was available (i.e., variants reported as 'no data', 'not available' or 'not yet reviewed'), variants were classified in accordance with the ENIGMA criteria. Specifically, truncating variants (i.e., nonsense, frameshift insertions or deletions and variants occurring in consensus splice 
Table 4. Variants of unknown significance ${ }^{a}$ in BRCA1 and BRCA2 identified by Hi-Plex targeted sequencing, in 426 women affected with breast or ovarian cancer in south-west Poland and west Ukraine.

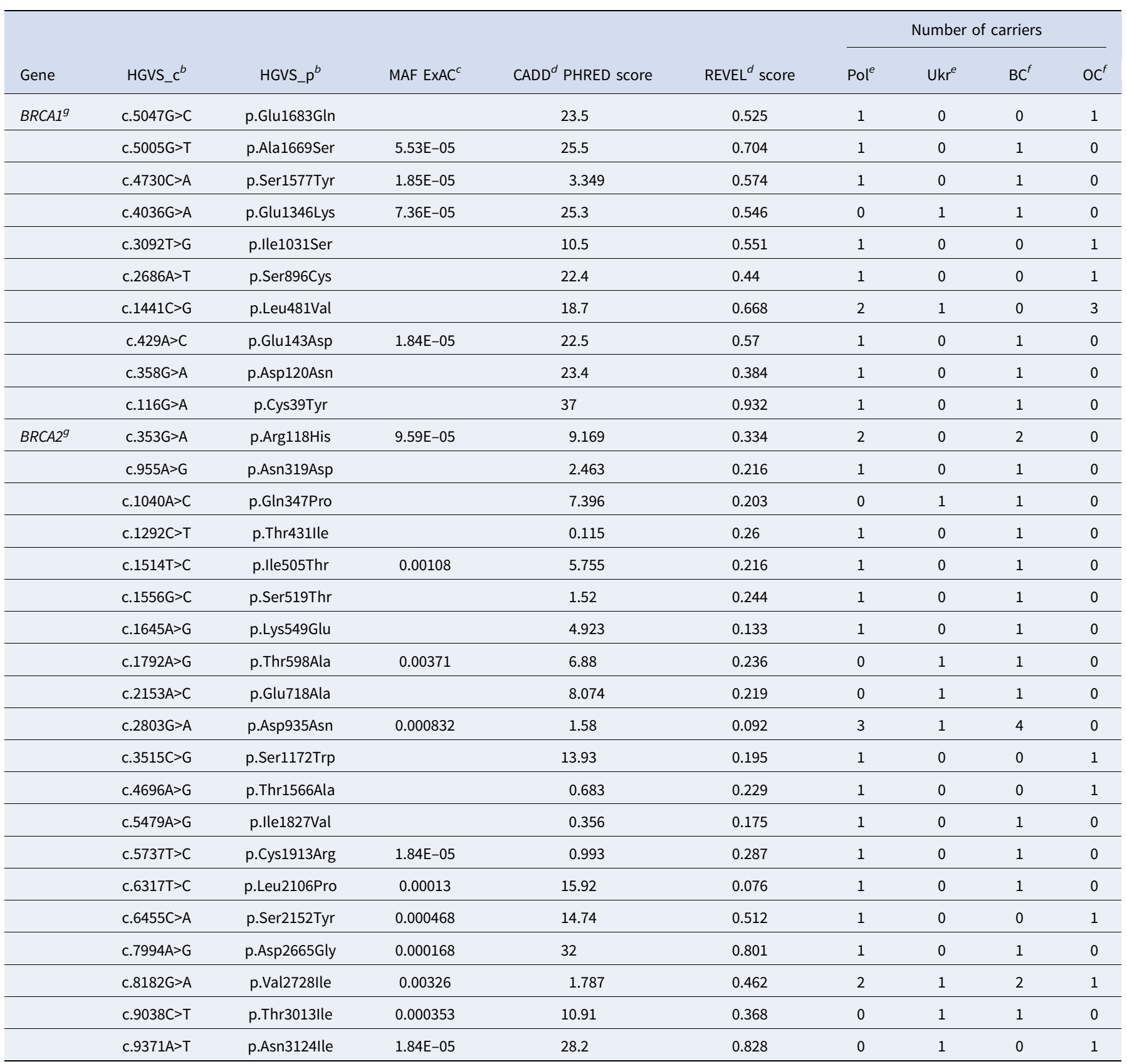

${ }^{a}$ Missense substitutions in BRCA1 and BRCA2 that are present at less than $1 \%$ in ExAC, that have not been reviewed yet or are classified as variants of unknown significance on BRCA Exchange by the Evidence-based Network for the Interpretation of Germline Mutant Alleles (ENIGMA) expert panel (Spurdle et al., 2012).

${ }^{b}$ Variant nomenclature based on +1 as A of ATG start codon, according to the Human Genome Variation Society (HGVS), HGVS_c for coding DNA and HGVS_P for protein variants.

${ }^{c}$ ExAC non-Finnish European population minus The Cancer Genome Atlas (Lek et al., 2016).

${ }^{d}$ CADD (Kircher et al., 2014); REVEL (Ioannidis et al., 2016).

${ }^{e} \mathrm{Pol}=$ Polish; Ukr= Ukrainian.

${ }^{f} \mathrm{BC}=$ breast cancer; $\mathrm{OC}=$ ovarian cancer.

${ }^{g}$ Transcript sequences are BRCA1: NM_007294.3 and BRCA2: NM_00059.3.

junctions) were classified as pathogenic/likely pathogenic (P/LP). Missense substitutions that had not been reviewed by ENIGMA were classified as variants of unknown significance (VUS).

\section{Results}

Targeted sequencing identified a total of 31 and 18 women as carriers of a $\mathrm{P} / \mathrm{LP}$ variant in $B R C A 1$ and $B R C A 2$, respectively
(Tables 2 \& 3). The clinical characteristics of these women are available in Supplementary Table S1.

The $49 \mathrm{P} / \mathrm{LP}$ variants with a variant allele fraction $>0.2$ and total depth $\geq 10 \times$ were verified by Sanger sequencing, except for the case that was identified as carrying the BRCA1 variant NM_007294.3: c. $4357+1 \mathrm{G}>\mathrm{C}$, as there was insufficient DNA available. That position was covered by 1302 reads, and the variant allele fraction was 0.53 and thus highly unlikely to be a sequencing artefact. 
The Polish women participating in this study were previously genotyped for mutations in BRCA1 and BRCA2. The prevalence of BRCA1 founder mutations (c.5266dup, c.181T $>\mathrm{G}$, c.4035del, c.68_69del) identified prior to this study was $10.1 \%(34 / 337)$ (Tables $2 \& 3$ ). Targeted sequencing did not identify any additional carrier of the mutations previously tested. We detected five carriers of a non-founder P/LP BRCA1 variant and eight carriers of a P/LP BRCA2 variant (3.9\% combined prevalence of non-founder mutations) (Table 3 ).

Among the Ukrainian women, the prevalence of founder mutations was $13 \%(16 / 123)$ (Table 3$)$. We identified 11 carriers of BRCA1:c.5266dup, 3 carriers of BRCA1:c.181T $>\mathrm{G}$ and 2 carriers of BRCA1:c.68_69del. We did not observe BRCA1: c.4035del in the Ukrainian cohort. There were 10/123 carriers of a P/LP variant in BRCA1 and 10/123 carriers of a P/LP variant in BRCA2 (16.3\% combined prevalence of non-founder P/LP variants) (Table 3). BRCA1:c.5030_5033del was observed in three unrelated women with breast cancer from Ukraine, all of whom having a family history of cancer (one had a sister with breast cancer and two had mothers with ovarian cancer) (Supplementary Table S1).

Targeted sequencing also identified 11 and 27 rare missense substitutions in BRCA1 and BRCA2, respectively, which are currently classified as VUS (Table 4).

\section{Discussion}

The overall prevalence of P/LP BRCA1 and BRCA2 variants in the Polish women in this study was $13.9 \%$ (47/337) (Table 3). Of these, over a quarter were non-founder mutations $(27.7 \%, 13 / 47)$. Our findings are consistent with a recent report by Kowalik et al., who screened for BRCA1 and BRCA2 in Polish women who qualified for genetic testing and identified $161 \mathrm{P} / \mathrm{LP}$ variants, $64 \%$ $(103 / 161)$ of which were founder mutations and $24.8 \%(40 / 161)$ of which were non-founder P/LP variants (Kowalik et al., 2018).

To the best of our knowledge, our study is the first to report the mutation screening of the complete coding regions of BRCA1 and $B R C A 2$ in Ukrainian women affected with breast and/or ovarian cancer. In the Ukrainian women, the overall prevalence of P/LP $B R C A 1$ and BRCA2 variants was $29.3 \%(36 / 123)$ (Table 3$)$. There was no difference in the proportion of founder and non-founder mutations $(44.4 \%, 16 / 36$, and $55.5 \%, 20 / 36$, respectively).

The higher overall prevalence of P/LP variants observed in the Ukrainian participants $(29.3 \%$ versus $13.9 \%$ in the Polish participants) is likely due to differences in selection criteria. Among the Ukrainian women, 99\% (122/123) of participants met the criteria for hereditary $(65 \%, 80 / 123)$ or familial cancer $(34 \%, 42 / 123)$, whereas the Polish cohort included a majority of sporadic cancers (54\%, 182/337) (Berliner et al., 2007). Hereditary and familial cancers in the Polish cohort accounted for only 38\% (127/337) and $8 \%(28 / 337)$ of all participants, respectively (Table 3$)$.

Overall, non-founder variants represented $43.9 \%(18 / 41)$, $62.5 \%(5 / 8)$ and $66.7 \%(2 / 3)$ of all P/LP variants observed in women affected with hereditary, familial and sporadic breast cancer, respectively (Table 3 ). In women affected with ovarian cancer, non-founder variants represented $14.3 \%(3 / 21)$ and $100 \%(3 / 3)$ of all $\mathrm{P} / \mathrm{LP}$ variants observed in hereditary and familial cancer, respectively. Expanding genetic testing beyond genotyping for founder variants has thus enabled us to identify 33 women carrying a clinically actionable variant who will be able to receive personalized clinical advice for themselves and their family. These results support the utility of comprehensive gene testing of BRCA1 and BRCA2 in Polish and Ukrainian patients, especially in women with hereditary and familial cancers.

In addition to $\mathrm{P} / \mathrm{LP}$ variants, our study identified 38/427 carriers $(7.7 \%)$ of rare missense variants of unknown clinical significance. Missense substitutions may result in variant proteins with functions ranging from normal to severely altered. Therefore, this group of variants is highly likely to be made up of variants with differing levels of associated risks (including none). There are substantial ongoing efforts by ENIGMA to classify VUS in BRCA1 and BRCA2 (Vallee et al., 2012). However, methods such as calibrated functional assays are essential for enabling variant classification, and we currently lack the evidence base from which to interpret and report most missense substitutions.

Our study applied an amplicon-based targeted sequencing methodology that is not designed to detect copy number variations (CNVs). The inherent nature of targeted sequencing poses substantial challenges for the detection of these variants. A number of software tools for CNV detection from targeted sequencing data have recently emerged to try to address this gap (Li et al., 2012; Ellingford et al., 2017; Kerkhof et al., 2017). However, they are developed for probe-based enrichment rather than amplicon-based methodologies and, to date, multiplex ligationdependent probe amplification remains the gold standard method for the clinical identification of such events.

\section{Conclusions}

Our results show that performing comprehensive genetic testing of $B R C A 1$ and BRCA2 instead of testing for founder mutations only will be highly valuable in Poland and Ukraine. Massively parallel sequencing is an effective way of performing comprehensive genetic testing of BRCA1 and BRCA2 that will increase the detection rate of clinically actionable variants and thus enhance risk assessment and management for these women and their families.

Supplementary material. The supplementary material for this article can be found at https://doi.org/10.1017/S0016672320000075

Acknowledgements. We would like to thank Magdalena Puniach for her assistance with translating the Polish data.

Author contributions. TN-D contributed to the study design, performed the sequencing data analyses and drafted the manuscript. PK, MMS, RS HA, AgS, KP and NK collected the study sample and provided clinical information. JAS and DT contributed to the processing of the sequencing data. FH contributed to the genetic testing. DJP and BJP designed the Hi-Plex technology for the panel testing. PK, AlS and HT prepared the DNA samples. AM performed the genetic testing and validations. MCS and AM were responsible for the overall study design and contributed to the data analysis and drafting of the manuscript.

Financial support. This work was supported by the Australian National Health and Medical Research Council (APP1029974 and APP1074383) and by a Victorian Life Sciences Computation Initiative grant (number VR0182) on its Peak Computing Facility, an initiative of the Victorian Government. TN-D is a Career Development Fellow of the National Breast Cancer Foundation (Australia, ECF-17-001). BJP is the recipient of a Victorian Health and Medical Research Fellowship (Australia). MCS is a National Health and Medical Research Council (Australia) Senior Research Fellow. AM was supported by a Research Fellowship within 'UR - modernity and future of region' from the European Social Fund, Human Capital, National Cohesion Strategy (contract no. UDA-POKL.04.01.01-00-068/ 10-00).

Conflicts of interest. The authors declare that they have no conflicts of interest. 
Availability of data and material. The datasets used and/or analysed during the current study are available from the corresponding author on reasonable request.

\section{References}

Berliner, J.L., Fay, A.M. \& Practice Issues Subcommittee of the National Society of Genetic Counselors' Familial Cancer Risk Counseling Special Interest Group (2007). Risk assessment and genetic counseling for hereditary breast and ovarian cancer: recommendations of the National Society of Genetic Counselors. Journal of Genetic Counseling 16, 241-260.

Brozek, I., Cybulska, C., Ratajska, M. et al. (2011). Prevalence of the most frequent BRCA1 mutations in Polish population. Journal of Applied Genetics 52, 325-330.

Cybulski, C., Kluzniak, W., Huzarski, T. et al. (2019). The spectrum of mutations predisposing to familial breast cancer in Poland. International Journal of Cancer 145, 3311-3320.

Ellingford, J.M., Campbell, C., Barton, S. et al. (2017). Validation of copy number variation analysis for next-generation sequencing diagnostics. European Journal of Human Genetics 25, 719-724.

Gorski, B., Cybulski, C., Huzarski, T. et al. (2005). Breast cancer predisposing alleles in Poland. Breast Cancer Research and Treatment 92, 19-24.

Hamel, N., Feng, B.J., Foretova, L. et al. (2011). On the origin and diffusion of BRCA1 c.5266dupC (5382insC) in European populations. European Journal of Human Genetics 19, 300-306.

Ioannidis, N.M., Rothstein, J.H., Pejaver, V. et al. (2016). REVEL: an ensemble method for predicting the pathogenicity of rare missense variants. American Journal of Human Genetics 99, 877-885.

Janavicius, R., Rudaitis, V., Feng, B.J. et al. (2013). Haplotype analysis and ancient origin of the BRCA1 c.4035delA Baltic founder mutation. European Journal of Medical Genetics 56, 125-130.

Kaufman, B., Laitman, Y., Gronwald, J., Lubinski, J. and Friedman, E. (2009). Haplotype of the C61 G BRCA1 mutation in Polish and Jewish individuals. Genetic Testing and Molecular Biomarkers 13, 465-469.

Kerkhof, J., Schenkel, L.C., Reilly, J. et al. (2017). Clinical validation of copy number variant detection from targeted next-generation sequencing panels. Journal of Molecular Diagnostics 19, 905-920.

Kircher, M., Witten, D.M., Jain, P., O'Roak, B.J., Cooper, G.M. and Shendure, J. (2014). A general framework for estimating the relative pathogenicity of human genetic variants. Nature Genetics 46, 310-315.

Kowalik, A., Siolek, M., Kopczynski, J. et al. (2018). BRCA1 founder mutations and beyond in the Polish population: a single-institution BRCA1/2 next-generation sequencing study. PLoS One 13, e0201086.

Kuchenbaecker, K.B., Hopper, J.L., Barnes, D.R. et al. (2017). Risks of breast, ovarian, and contralateral breast cancer for $B R C A 1$ and $B R C A 2$ mutation carriers. JAMA 317, 2402-2416.

Laitman, Y., Feng, B.J., Zamir, I.M. et al. (2013). Haplotype analysis of the 185delAG BRCA1 mutation in ethnically diverse populations. European Journal of Human Genetics 21, 212-216.

Lai, Z., Markovets, A., Ahdesmaki, M. et al. (2016). VarDict: a novel and versatile variant caller for next-generation sequencing in cancer research. Nucleic Acids Research 44, e108.

Lek, M., Karczewski, K.J., Minikel, E.V. et al. (2016). Analysis of proteincoding genetic variation in 60,706 humans. Nature 536, 285-291.

Li, H. and Durbin, R. (2009). Fast and accurate short read alignment with Burrows-Wheeler transform. Bioinformatics 25, 1754-1760.

Li, J., Lupat, R., Amarasinghe, K.C. et al. (2012). CONTRA: copy number analysis for targeted resequencing. Bioinformatics 28, 1307-1313.

McLaren, W., Gil, L., Hunt, S.E. et al. (2016). The Ensembl variant effect predictor. Genome Biology 17, 122.

Myszka, A., Nguyen-Dumont, T., Karpinski, P. et al. (2018). Targeted massively parallel sequencing characterises the mutation spectrum of PALB2 in breast and ovarian cancer cases from Poland and Ukraine. Familial Cancer $17,345-349$.

Nguyen-Dumont, T., Hammet, F., Mahmoodi, M. et al. (2015). Mutation screening of PALB2 in clinically ascertained families from the Breast Cancer Family Registry. Breast Cancer Research and Treatment 149, 547-554.

Paila, U., Chapman, B.A., Kirchner, R. and Quinlan, A.R. (2013). GEMINI: integrative exploration of genetic variation and genome annotations. PLoS Computational Biology 9, e1003153.

Spurdle, A.B., Healey, S., Devereau, A. et al. (2012). ENIGMA-evidencebased network for the interpretation of germline mutant alleles: an international initiative to evaluate risk and clinical significance associated with sequence variation in BRCA1 and BRCA2. Human Mutation 33, $2-7$.

Vallee, M.P., Francy, T.C., Judkins, M.K. et al. (2012). Classification of missense substitutions in the BRCA genes: a database dedicated to Ex-UVs. Human Mutation 33, 22-28. 